\title{
An overflow of... what else but metabolism!
}

\author{
Royston Goodacre
}

Published online: 23 February 2010

(C) Springer Science+Business Media, LLC 2010

Welcome to the Sixth Volume of Metabolomics!

It has been an exciting five years and last year we received our first impact factor from Thomson Reuters, calculated as 3.254. This is an excellent result and one I am delighted with. Congratulations to all our authors for sending us their most exciting and novel work, as well as top-notch reviews and tutorials. The aim is to improve this year-on-year by continuing to publish high quality, interesting and timely articles. Unfortunately for some papers this will result in rejection: our current reject rate is $c a$. $40 \%$.

I hope you will have noticed that the front cover of Metabolomics has undergone a transformation. It was decided at our recent Editorial Board meeting, held at last year's highly successful Metabolomics Society 5th International Meeting in Edmonton, Canada, that the Journal cover will change to allow for the inclusion of some artwork from an article featured in that current issue; this will be scientifically driven. Our first front cover is from Orešič's group (Yetukuri et al. 2010) on the functional prediction of yet to be identified lipids using supervised learning methods. Congratulations to the group for being featured on our first new front cover!

You may also notice that the Journal is a little larger than normal and this reflects that the field is continuing to expand. Figure 1 shows this growth is currently a healthy exponential. The journal will aim to grow with the field and so we are currently publishing $c a$. 14 articles per issue this year, and will consider expanding to six issues per annum

R. Goodacre $(\bowtie)$

School of Chemistry and Manchester Interdisciplinary

Biocentre, University of Manchester, 131 Princess Street,

Manchester M1 7DN, UK

e-mail: Roy.Goodacre@manchester.ac.uk soon. This also necessitates that we reduce the number of printed figures and tables. These will generally be limited to six in total, although authors are of course encouraged to submit supporting supplementary information that will appear electronically on the Journal's web site rather than in print (this includes metabolomics data).

Metabolomics research is often used as a discovery tool, or what may be termed hypothesis generating, in order to find out which metabolites are indicative of disease, therapeutic progression, or adverse drug reactions. This approach is at the cutting edge trisection of biological sciences, analytical chemistry, and chemometrics. A nice of example of this work was demonstrated last year by Sreekumar et al. (2009) who showed that sarcosine is a predictive biomarker for the differentiation of aggressive prostate cancer progression from non-invasive organ-confined disease. This discovery is exciting as this may, after validation in a large epidemiological study, become a useful prognostic marker for this disease's aggressiveness; moreover, this may also allow new insight into the pathophysiological process that occurs during the metastatic disease phase of prostate cancer.

For the above study to be successful rigorous statistical processing of the data was needed, and this these authors did achieve. In biomarker research I often think of the phrase first uttered by the 19th Century British Prime Minister Benjamin Disraeli_- "There are three kinds of lies: lies, damn lies, and statistics!" For the hypothesis generation phase to be successful it is important to avoid false discoveries and this we were reminded of in the comprehensive review by Broadhurst and Kell (2006) in this Journal. The authors are to be congratulated not only for an insightful argument as to why most findings in science are incorrect and what to do to avoid this, but also for being the first paper in Metabolomics to gain more than 50 


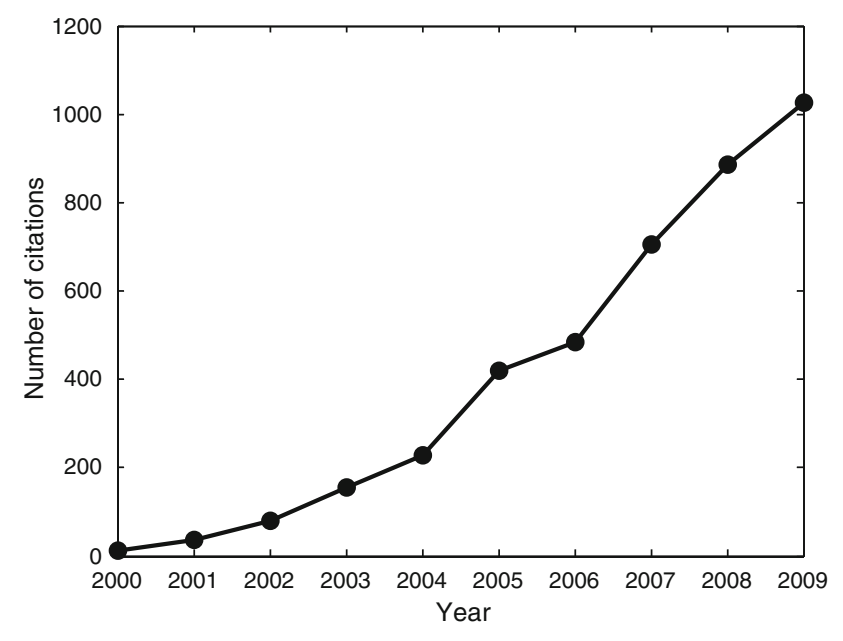

Fig. 1 Number of papers published per annum using the search term "metabolom" or "metabonom" in ISI Web of Knowledge (Thomson Reuters)

citations (source: ISI Web of Knowledge (Thomson Reuters); Dec 2009).

As well as publishing regular articles and reviews Metabolomics has also published special issues on Plant Metabolomics (see Vol 3(3)) and Environmental Metabolomics (see Vol 5(1)) and I am very grateful to George Harrigan and Mark Viant for acting as guest editors. I am also grateful to Oliver Fiehn and colleagues for submitting the Metabolomics Standards Initiative (MSI) proposed minimum/standard reporting requirements for metabolomics experiments (see Vol 3(3)). These are essential reading for all metabolomics scientists and these articles help set the scene in terms of what the MSI thinks needs to be reported. I would therefore encourage all authors to make their submissions as MSI compliant as possible with respect to experimental conditions, chemical analysis and data processing. In addition, as many metabolites have multiple synonyms; for example, for fructose there are 21 synonyms in PubChem (CID 439709) including the more obvious D-fructose but rather bizarre 'beta-fruit sugar', so using different names is likely to lead to confusion. Authors are therefore encouraged that when metabolite identities are reported that they are accompanied with a unique chemical identifier. There are a few to choose from (see Sumner et al. 2007), and perhaps ChEBI (http://www. ebi.ac.uk/chebi) is a good place to start as it has a tree-like structure; so for fructose one would report CHEBI:28757 and this is then linked to its isomeric forms (L-fructose is CHEBI:28120 and D-fructose is CHEBI:15824).

The first article in this issue is a tutorial review by Smilde et al. (2010) on dynamic metabolomic data analysis and we aim to publish regular reviews and tutorials. You are encouraged to email an outline of an intended review to me or any of the Editorial Board for consideration.

Finally, Metabolomics also has a twitter presence (http:// twitter.com/metabolomics) and there are regular updates on the Journal tweeted there.

I hope you enjoy this issue and continue to read and send your best work to Metabolomics.

\section{References}

Broadhurst, D. I., \& Kell, D. B. (2006). Statistical strategies for avoiding false discoveries in metabolomics and related experiments. Metabolomics, 2, 171-196.

Smilde, A. K., Westerhuis, J. A., Hoefsloot, H. C. J., Bijlsma, S., Rubingh, C. M., Vis, D. J., et al. (2010). Dynamic metabolomic data analysis: a tutorial review. Metabolomics, 6(1). doi: 10.1007/s11306-009-0191-1.

Sreekumar, A., Poisson, L. M., Rajendiran, T. M., Khan, A. P., Cao, Q., $\mathrm{Yu}$, J., et al. (2009). Metabolomic profiles delineate potential role for sarcosine in prostate cancer progression. Nature, 457, 910-914.

Sumner, L. W., Amberg, A., Barrett, D., Beger, R., Beale, M. H., Daykin, C., et al. (2007). Proposed minimum reporting standards for chemical analysis. Metabolomics 3, 211-221.

Yetukuri, L., Tikka, J., Hollmén, J., \& Orešič, M. (2010). Functional prediction of unidentified lipids using supervised classifiers. Metabolomics, 6(1). doi:10.1007/s11306-009-0179-x. 